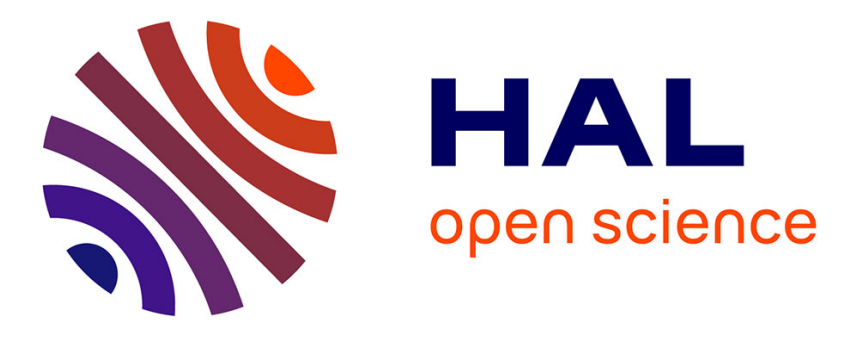

\title{
Optimal Size of Nanoparticles for Magnetic Hyperthermia: A Combined Theoretical and Experimental Study
}

Boubker Mehdaoui, Anca Meffre, Julian Carrey, Sebastien Lachaize, Lise-Marie Lacroix, Michel Gougeon, Bruno Chaudret, Marc Respaud

\section{To cite this version:}

Boubker Mehdaoui, Anca Meffre, Julian Carrey, Sebastien Lachaize, Lise-Marie Lacroix, et al.. Optimal Size of Nanoparticles for Magnetic Hyperthermia: A Combined Theoretical and Experimental Study. Advanced Functional Materials, 2011, 21 (23), pp.4573-4581. 10.1002/adfm.201101243 . hal01952258

\section{HAL Id: hal-01952258 \\ https://hal.science/hal-01952258}

Submitted on 12 Dec 2018

HAL is a multi-disciplinary open access archive for the deposit and dissemination of scientific research documents, whether they are published or not. The documents may come from teaching and research institutions in France or abroad, or from public or private research centers.
L'archive ouverte pluridisciplinaire HAL, est destinée au dépôt et à la diffusion de documents scientifiques de niveau recherche, publiés ou non, émanant des établissements d'enseignement et de recherche français ou étrangers, des laboratoires publics ou privés. 
DOI: 10.1002/adfm.((please insert DOI)

Submitted to

\section{Optimal size of nanoparticles for magnetic hyperthermia:}

\section{A combined theoretical and experimental study}

By B. Mehdaoui, A. Meffre, J. Carrey*, S. Lachaize, L. M. Lacroix, M. Gougeon, B. Chaudret, and M. Respaud

[*] Dr B. Mehdaoui, Dr A. Meffre, Dr J. Carrey Corresponding Author, Dr S. Lachaize, Dr L. M. Lacroix, Prof B. Chaudret, Prof M. Respaud

Université de Toulouse; INSA; UPS; LPCNO (Laboratoire de Physique et Chimie des NanoObjets), 135 avenue de Rangueil, F-31077 Toulouse, France and

CNRS; UMR 5215 ; LPCNO, F-31077 Toulouse, France

E-mail: ((julian.carrey@insa-toulouse.fr))

Dr. M. Gougeon

Institut CARNOT - CIRIMAT - UMR 5085, Bâtiment 2R1, 118 route de Narbonne F-31062 Toulouse, France

Keywords: hyperthermia, magnetic nanoparticles, iron, hysteresis loop calculation

Progresses in the prediction and optimization of the heating of magnetic nanoparticles in an alternative magnetic field are highly desirable for their application in magnetic hyperthermia. Here a model system consisting of metallic iron nanoparticles with a size ranging from 5.5 to $28 \mathrm{~nm}$ is extensively studied. Different regimes as a function of the nanoparticles size are evidenced: single-domain superparamagnetic, single-domain ferromagnetic and multi-domain. Ferromagnetic single-domain nanoparticles are the best candidates and display the highest specific losses reported in the literature so far $\left(11.2 \pm 1 \mathrm{~mJ} \mathrm{~g}^{-1}\right)$. Measurements are analysed using state-of-the-art analytical formula and numerical simulations of hysteresis loops. Several features expected theoretically are observed for the first time experimentally: i) the correlation between the nanoparticle diameter and their coercive field ii) the correlation between the amplitude of the coercive field and the losses iii) the variation of the optimal size with the amplitude the magnetic field. None of these features are predicted by the linear response theory -generally used to interpret hyperthermia experiments- but are a natural 
Submitted to 14 A R

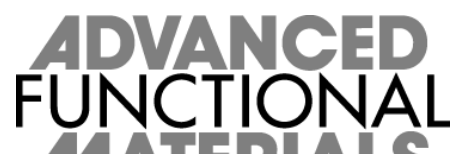

consequence of theories deriving from the Stoner-Wohlfarth model; they also appear clearly in numerical simulations. These results open the path to a more accurate description, prediction and analysis of magnetic hyperthermia.

\section{Introduction}

Magnetic hyperthermia $(\mathrm{MH})$ is a promising therapeutic method based on the use of magnetic nanoparticles (MNPs). MH is a two step procedure: MNPs are first injected into the tumour; the patient is then immersed in an alternating magnetic field with a frequency $f$ and an amplitude $\mu_{0} H_{\max }$ appropriately chosen. Due to the excitation of the MNPs, the temperature of the tumour rises. This temperature increase improves the efficiency of chemotherapy $\left(\mathrm{T} \sim 42-45^{\circ} \mathrm{C}\right)$ or can even directly kill the tumor cells by necrosis $\left(\mathrm{T}>50^{\circ} \mathrm{C}\right)[1,2,3,4,5,6]$.

When applying an alternative magnetic field to MNPs, the area of their magnetic hysteresis loop $A$ corresponds to a dissipated energy, which is used in MH. The power generated by the MNPs is evaluated by this specific area $A$ (specific losses) or, more commonly used, by their specific absorption rate (SAR), the two parameters being linked by the equation $S A R=A f$. Exploiting this effect in oncology requires biocompatible MNPs with large heating power, thus one of the challenges in this topic is to maximize SAR values. The SAR being an increasing function of $f$ and $\mu_{0} H_{\max }$, one could think of increasing these parameters. However, human body cannot be exposed to alternating magnetic field of large $\mu_{0} H_{\max } f$ product, due to medical limitation [7]. Therefore, optimizing the MNPs requires optimizing their SAR at finite and specific values of the applied frequency and magnetic field. Typical values used in medical treatments so far are $100 \mathrm{kHz}$ and $20 \mathrm{mT}$ [8].

For experimentalists, there are three main ways to optimize the SAR. The first one is to increase the saturation magnetization $M_{\mathrm{S}}$ of MNPs since the maximum SAR achievable is directly proportional to $M_{\mathrm{S}}$. The second way is to optimize the anisotropy of the MNPs since the importance of this parameter has been recently shown [9]. These first two points help in determining the optimal materials. Finally, for a given material, the next major parameter is the MNP size. An experimental study of this parameter requires synthesizing monodisperse MNPs with a varying mean size and studying the size-dependence of SAR. This has been realised in a few articles centred on the properties of iron oxide MNPs [10, 11, 12, 13, 14]. 


\section{Submitted to}

In the previous studies, when a quantitative analysis was tempted, $[11,12,13,14]$ the linear response theory (LRT) was always used, which is a major problem. Indeed, this theory has a restricted domain of validity [9], but the studies mentioned above have at some point used LRT out of this domain, which casts doubt on the results obtained (on this issue, see [15]). For a rigorous analysis of experimental data, theories derived from the StonerWohlfarth model in their domain of validity should be used in complement to LRT $[9,16]$. Out of the domain of validity of these two models, only numerical simulations can confidently calculate the SAR of single-domain MNPs $[9,17]$. However, such simulations have never been applied so far for a direct comparison with experiments.

The aim of the present article is precisely to compare experiments performed on a model system consisting of Fe MNPs, with a tuneable mean size ranging from 5.5 to $28 \mathrm{~nm}$, with state-of-the-art methods of analysis consisting of numerical simulations and adequate theories. Iron is a material with a weak anisotropy and a high $M_{\mathrm{S}}$; its magnetic properties are less sensitive to unwanted size and surface effects such as dead layers than its oxide counterparts due to its metallic character. The synthesized Fe MNPs studied here are highly crystalline and display a $M_{\mathrm{S}}$ value close to the bulk one in the whole range of size studied. Thus, these MNPs constitute a perfect system for a fine study on the influence of the MNP size on $\mathrm{MH}$.

Here we show that the magnetic-field dependence of the SAR and its amplitude strongly depends on the MNP size. In particular the specific losses are maximized for MNPs in a narrow range of diameters, and reach unprecedented values. Behaviour typical of singledomain MNPs in the superparamagnetic regime, in the ferromagnetic regime and of multidomain MNPs are observed. Moreover, several features in the size-dependence of MH are reported here for the first time and can be well understood using theories derived from the Stoner-Wohlfarth model and numerical simulations. An original method for the quantitative analysis of $\mathrm{MH}$ is proposed and applied to our experimental results, permitting their fine analysis. Thus, this article confirms the limitation of LRT theory to explain experimental results on systems of interest for MH. It evidences that the use of suitable theories opens the path to more accurate description, prediction and analysis of $\mathrm{MH}$.

\section{Experimental results}




\subsection{Synthesis and characterisations}

Submitted to

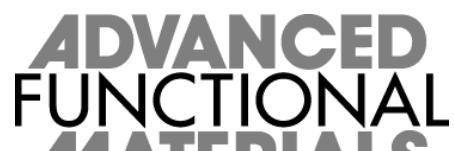

MATERIALS

Our group has been investigating the elaboration of pure metallic iron(0) nanoparticles using an organometallic approach for about 10 years. $[18,19,20]$ This approach is based on the decomposition of an iron source in a reductive medium in the presence of surfactants. By varying the reactants (iron source, surfactants) and the experimental conditions (concentration, temperature or pressure), we covered a large panel of size and shape for iron(0) nanoparticles. In this article, the iron sources were either the iron dimer $\left\{\mathrm{Fe}\left[\mathrm{N}\left(\mathrm{SiMe}_{3}\right)_{2}\right]_{2}\right\}_{2}$ or the ultra-small $\mathrm{Fe}(0) \mathrm{NPs}$ whose preparation has already been reported elsewhere.[20] The surfactant system can be one of the two following combinations: palmitic acid (PA) / hexadecylamine (HDA) or hexadecylamonium chloride (HDA·HCl) / HDA. In all the cases, the reactants were reacted in mesitylen, under a reductive atmosphere of dihydrogen at $150^{\circ} \mathrm{C}$ for 2 days. Depending on the conditions, we forced different growth mechanisms to take place in order to change the morphology of the final NPs. Thus, the spherical NPs of samples 1 and 2 were formed thanks to an isotropic growth regulated by the PA/HDA ratio. Increasing the quantity of carboxylic acid over the amine led to a size increase because the growth is then favoured over the nucleation.[19] The same trend was observed for samples 3 to 7 that were prepared with the ammonium chloride $\mathrm{HDA} \cdot \mathrm{HCl}$ at various concentrations.[21] Samples 3 and 5 were prepared from the $\left\{\mathrm{Fe}\left[\mathrm{N}\left(\mathrm{SiMe}_{3}\right)_{2}\right]_{2}\right\}_{2}$ iron source whereas samples $\mathbf{4}$, $\mathbf{6}$ and 7 were formed from ultra-small $\mathrm{Fe}(0) \mathrm{NPs}$. Changing the iron source nature helped us to produce different samples by playing with the different reduction kinetics. Tuning the experimental conditions finally allowed us to produce 7 samples of different shapes and mean sizes displaying a magnetization close to the bulk value. One main difference between the spherical and the cubic NPs is their aggregation in solution. Due to the shape and the larger mean size, the samples with cubic NPs are not colloids: the NPs self-organize into large superlattices. The latter are strongly stabilized by Van der Waals interactions between the alkyl chains of the surfactants at the surface of the NPs, and by magnetic interaction between the ferromagnetic NPs. These two contributions prevent any good dispersion of the NPs in compatible solvents.

Transmission electron microscopy (TEM) characterisations of the samples are shown in Figure 1. Magnetic parameters extracted from SQUID measurements (see Supplementary Information) are summarized in Table 1. For all samples, except sample 4, the saturation magnetization per unit mass $\sigma_{\mathrm{S}}$ is close to the bulk Fe magnetization $\left(212 \mathrm{~A} \mathrm{~m}^{2} \mathrm{~kg}^{-1}\right)$. 


\section{Submitted to}

\subsection{Magnetic hyperthermia measurements}

$\mathrm{MH}$ measurements at $54 \mathrm{kHz}$ were performed on a specially designed frequencyadjustable electromagnet [22]. This setup has been recently improved and allows us to work with a magnetic field up to $\mu_{0} H_{\max }=60 \mathrm{mT}$ for frequencies $f$ in the range $5-100 \mathrm{kHz}$. Measurements at $274 \mathrm{kHz}$ were performed using the coil of an induction oven, with a magnetic field up to $\mu_{0} H_{\max }=73 \mathrm{mT}$, similarly to experiments described in Ref. [23] (see Experimental Section). Preliminary results on the $\mathrm{MH}$ properties of samples $\mathbf{1}$ and $\mathbf{4}$ were published in Refs [24] and [23], respectively.

The SAR dependence as a function of magnetic field at frequencies $f=54 \mathrm{kHz}$ and $f=$ $274 \mathrm{kHz}$ for samples $\mathbf{1 - 7}$ are shown in Figures $\mathbf{2 a}$ and $\mathbf{2 b}$ respectively. For sample $\mathbf{1}(d=5.6$ $\mathrm{nm}$ ) the magnetic field dependence of the SAR follows a power law with an exponent of 2.8 and 3.1 at frequencies $f=54 \mathrm{kHz}$ and $274 \mathrm{kHz}$ respectively. Their behaviour has been analysed in details in a previous article and is typical of MNPs in the superparamagnetic regime [24].

In samples 3-7 $(d=8.9-27.5 \mathrm{~nm})$, the SAR displays at $274 \mathrm{kHz}$ a saturation at large magnetic field (see Figure 2b), which is typical of the ferromagnetic regime [16]. At $54 \mathrm{kHz}$, the saturation is only visible on sample $3(d=8.9 \mathrm{~nm})$ because of the smaller maximum magnetic field available on this setup. On these samples the SAR shows a small amplitude at low magnetic field and a sharp increase above the coercive field of the MNPs, which is another feature of the ferromagnetic regime. The evolution of the coercive field with size is more clearly evidenced in Figure $\mathbf{2 c}$ and $\mathbf{2 d}$, where the normalized SAR values are shown for these samples. In samples 3-6, the coercive field increases with the size of the MNPs. It can be observed that the amplitude of the SAR at large magnetic field is directly related to the amplitude of the coercive field : the larger the coercive field, the larger the SAR. In sample 7 $(d=27.5 \mathrm{~nm})$, both the coercive field and the SAR displays an abrupt drop (see Figure 2a-d).

Another interesting way to plot the same experimental data is to display the size dependence of SAR for various applied magnetic fields. Such a plot is shown for the two frequencies in Figures 2e and 2f. It can be observed that, for each magnetic field, an optimal 
Submitted to

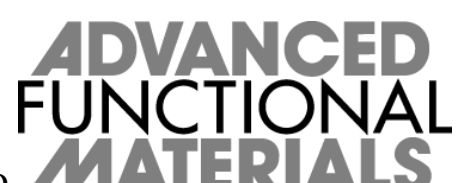

diameter $d_{\text {opt }}$ maximizes the amplitude of SAR. Interestingly, $d_{\text {opt }}$ is not constant but increases with the amplitude of the applied magnetic field.

Finally, the degree of optimization $\alpha$ achieved in a given MNPs system for an application in $\mathrm{MH}$ can be extracted from the SAR value using [9, 23]:

$$
S A R=4 \alpha \mu_{0} H_{\max } \sigma_{S} f
$$

where $\sigma_{S}$ is taken here as the bulk Fe value. $\alpha$ is a dimensionless parameter which characterizes the relative area of the hysteresis loops with respect to the ideal square $(\alpha=1)$. In Figures $2 \mathrm{~g}$ and $\mathbf{2 h}$ the evolution of $\alpha$ as a function of the magnetic field and MNP diameter is shown and its value at saturation is summarized in Table I.

For a possible application of such nanoparticles in medical applications, the $\mu_{0} H_{\max } f$ product is limited to be safe for the patient. We have performed hyperthermia measurements on the various samples at $35 \mathrm{kHz}$ and $57 \mathrm{mT}$ so as to keep the same $\mu_{0} H_{\max } f$ product as the one currently used at the Charité Hospital [8]. The evolution of SAR, $A$ and $\alpha$ with size are shown as Supporting Information. Sample $6(d=19.7 \mathrm{~nm})$ displays the largest SAR $=290$ $\mathrm{W} / \mathrm{g}$, corresponding to $A=8.3 \mathrm{~mJ} / \mathrm{g}$ and $\alpha=0.17$.

A few comments on the data shown in Figure 2 can be made. First, the SAR values measured on the optimized samples are very large. At $54 \mathrm{kHz}$, the maximum SAR $=427 \pm 42$ $\mathrm{W} \mathrm{g}^{-1}$ occurs for sample $6(d=19.7 \mathrm{~nm})$, while at $f=274 \mathrm{kHz}$, the maximum SAR = $3066 \pm 306 \mathrm{~W} \mathrm{~g}^{-1}$ occurs for sample $5(d=13.7 \mathrm{~nm})$. In the latter case, this corresponds to specific losses $A=11.2 \pm 1 \mathrm{~mJ} \mathrm{~g}^{-1}$, which are the highest reported in the literature so far. Interestingly, large values of $\alpha$ are obtained only for values of the magnetic field greater than $50 \mathrm{mT}$. In a previous article, we had theoretically shown that iron MNPs -due to the value of their anisotropy field around $50 \mathrm{mT}$ - should in principle be optimized nanoparticles only when magnetic fields of this order of magnitude are applied [9]. This is experimentally confirmed here. The largest $\alpha=0.19$ is observed in sample $5(d=13.7 \mathrm{~nm})$ under $\mu_{0} H_{\max }=43$ $\mathrm{mT}$ and $f=274 \mathrm{kHz}$. Though being the highest among our experiments, this value is still far 
Submitted to

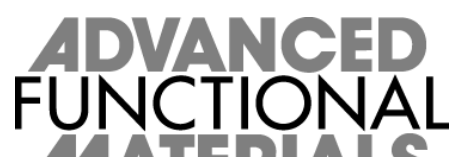

414 a

from the maximum value achievable in a system with randomly oriented anisotropy axis $\alpha=$ 0.39 [9]. Thus our system could be optimized further, as will be discussed in Section 3.2.2.

\section{Analysis}

\subsection{Qualitative analysis}

\subsubsection{Magnetic field dependence of SAR}

In this part, $\mathrm{MH}$ properties are calculated numerically without attempt to fit the experimental data. The results are then compared to experimental results for a qualitative analysis. The hysteresis loop area $A$ is calculated for an assembly of spherical, uniaxial MNPs with their anisotropy axis randomly distributed in space, as detailed in Ref. [9]. The magnetic parameters of bulk iron have been used $\left(M_{\mathrm{S}}=1700 \mathrm{kA} / \mathrm{m}\right.$, the effective anisotropy $K_{\text {eff }}=$ $4.8 \times 10^{4} \mathrm{~J} \mathrm{~m}^{-3}$ ). The other parameters are $T=300 \mathrm{~K}, f=100 \mathrm{kHz}$, and the frequency factor of the Néel-Brown relaxation time $\tau_{0}=5 \times 10^{-11} \mathrm{~s}$ As an illustration, the calculated hysteresis loops for $d=16 \mathrm{~nm}$ are shown in Figure 3a as a function of the maximum field applied.

In Figure 3b the evolution of $A$ with magnetic field for MNPs with a diameter $d$ ranging from $4 \mathrm{~nm}$ to $24 \mathrm{~nm}$ is shown. In Figure 3c normalized data illustrating more clearly the evolution of the curve shape for the different diameters are shown. It can be seen that the shape of the $A\left(\mu_{0} H_{\max }\right)$ evolves significantly with the diameter. For the smallest MNPs $(d=4$ $\mathrm{nm}), A$ shows a square dependence with the magnetic field in agreement with LRT for superparamagnetic MNPs [9,25]. For sizes up to $d=10 \mathrm{~nm}, A$ follows a power law, whose exponent vary with MNPs size [9]. For $d \geq 14 \mathrm{~nm}$, the shape of the curves is characteristic of the ferromagnetic regime: the losses are very weak at low magnetic field followed by an increase around the coercive field and finally a progressive saturation $[16,26]$.

The comparison between numerical calculations (Figure 3) and experiments (Figure 2) shows a qualitative agreement on three main features: i) the difference of behaviour between the superparamagnetic regime and the ferromagnetic one, the former displaying small SAR values and no saturation at large magnetic field, ii) the correlation between the amplitude of the coercive field and the amplitude of SAR for MNPs in the ferromagnetic regime and iii) 
Submitted to

the increase of SAR with the MNP diameter at high magnetic field. The main point of discrepancy comes from the sample $7(d=27.5 \mathrm{~nm})$, which displays an abrupt drop of coercive field and SAR. This drop in coercive field can be explained by the transition toward a multi-domain state of the MNP magnetization. Indeed, a transition from single-domain to vortex configuration has been calculated by micromagnetic simulations for Fe particles of roughly $d=26 \mathrm{~nm}$ [27]. Moreover, magnetic holography measurements performed on a 30 $\mathrm{nm}$ nanocube, similar to the ones composing this sample, have evidenced a vortex state of the magnetic configuration [28].

\subsubsection{Optimal size}

To study the optimal diameter $d_{\mathrm{opt}}$ or the optimal volume $V_{\mathrm{opt}}$ for $\mathrm{MH}$, the evolution of $A$ as a function of the diameter at a fixed $\mu_{0} H_{\max }$ value must be evaluated. In order to do so, hysteresis loops are calculated numerically for various sizes, the parameter values being chosen as previously. Figure 4a shows an example for $\mu_{0} H_{\max }=20 \mathrm{mT}$-which is the magnetic field used during treatments [8]. For very small nanoparticles $\left(d<d_{\mathrm{opt}}\right)$, the hysteresis loop is almost reversible and specific losses $A$ almost null, MNPs are superparamagnetic. When the volume increases, the hysteresis loop progressively opens and displays a typical ferromagnetic shape. The area of the hysteresis loop reaches a maximum for $d=d_{\mathrm{opt}}=17.4 \mathrm{~nm}$, plotted in thick line. For $d>d_{\mathrm{opt}}$, the coercive field is too large compared to the applied magnetic field, so the magnetization of the MNPs cannot be switched, leading to minor loops only. Thus, the losses decrease with increasing size, and vanish at very large size.

The evolution of the area $A$ as a function of the MNP size is summarized in Figure $4 \mathbf{b}$ for various magnetic field amplitudes. The presence of an optimal diameter is clearly visible, permitting to plot the evolution of $d_{\mathrm{opt}}$ as a function of the magnetic field (Figure 4c). At very low magnetic field amplitude, the optimal diameter $d_{\mathrm{opt}}=11.7 \mathrm{~nm}$ is independent of the magnetic field amplitude. With strengthened $\mu_{0} H_{\max }, d_{\text {opt }}$ increases. The optimal size, derived here above from numerical calculations, can also be predicted analytically using two models. At low field, for $\mu_{0} H_{\max }<2.7 \mathrm{mT}$, LRT is valid, so the optimum volume is given by the following equation [9]:

$$
V_{o p t}=\frac{k_{B} T}{K} \ln \left(\pi f \tau_{0}\right)
$$


Submitted to

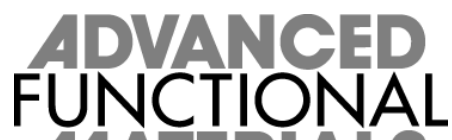

14ABRAS

The optimal volume is thus constant at low field, as represented in Figure 4c by the horizontal solid line.

In the ferromagnetic regime, LRT is no more valid, therefore theories derived from the Stoner-Wohlfarth model should be used instead. In this framework, one can have access to the optimal volume through the optimization of the coercive field $\mu_{0} H_{\mathrm{C}}$ which reads [9]:

$$
\mu_{0} H_{C}=0.48 \mu_{0} H_{K}\left(1-\kappa^{0.8}\right),
$$

where $H_{K}=\frac{2 K}{M_{S}}$ is the anisotropy field and

$$
\kappa=\frac{k_{B} T}{K V} \ln \left(\frac{k_{B} T}{4 \mu_{0} H_{\max } M_{S} V f \tau_{0}}\right) .
$$

Note that Equation (3) is only valid when $\kappa<0.7$. The maximum value for $A$ is obtained for an optimal coercive field, the value of which is slightly below the applied magnetic field [6] : $\mu_{0} H_{C}=(0.81 \pm 0.04) \mu_{0} H_{\max }$.

Using Equations (3) and (5), one can then deduce the optimal volume and thus, the optimal diameter. The result of this resolution is shown in Figure $4 \mathbf{c}$ as a dashed line in the domain of validity of Equation (3). This figure illustrates that the evolution of the optimal size at large magnetic field is a natural consequence of theories derived from the Stoner-Wohlfarth model.

Here again, comparing Figure $4 \mathrm{~b}$ with Figure 2e-f shows that this feature is observed experimentally, since the optimal diameter increases with the magnetic field. This evolution comes with an increase of SAR. This is the first time that this feature is observed experimentally.

\subsection{Quantitative analysis}

\subsubsection{Method and hypothesis}

The experimental and theoretical results presented above are in good qualitative agreement, insofar as all the main features predicted theoretically are observed experimentally. However, there are some quantitative disagreements on the values of the losses, on the optimal diameters and on the coercive field values. These differences cannot be explained only by the frequency used. For instance, a coercive field of $c a .10 \mathrm{mT}$ is 
Submitted to

determined numerically for $14 \mathrm{~nm}$ MNPs at $100 \mathrm{kHz}$ (cf Figure 3b) while values of $c a .40$ and $35 \mathrm{mT}$ are respectively obtained at 54 and $274 \mathrm{kHz}$ (cf Figure 2c-d). This discrepancy might result from several effects: different values of $\tau_{0}$ and/or $K_{\text {eff, influence of size distribution }}$ and/or of magnetic interactions. In order to determine the origin of this discrepancy, a quantitative analysis of the experimental results is required.

Due to the fact that the calculation time of a typical graph in Figures 3 or 4 is around one week, it is not reasonable to expect to vary both $\tau_{0}$ and $K_{\text {eff }}$ in numerical simulations until a good fit of experimental data is achieved. To make a quantitative analysis possible, a special method must be used and a few assumptions made. The method is the following: i) a "coercive field value deduced from $\mathrm{MH}$ experiments" $\mu_{0} H_{C H y p}$ is defined as the point of highest slope in $\operatorname{SAR}\left(\mu_{0} H_{\max }\right)$ functions. The interest of $\mu_{0} H_{C H y p}$ is that it can be calculated analytically for a sample composed of single-domain MNPs in the ferromagnetic regime. ii) Thus our analysis is restricted to the samples 3-6 $(d=8.9-19.7 \mathrm{~nm})$, given that these samples match these requirements. iii) We assume that these four samples have the same $K_{\text {eff }}$ value, which it is not unreasonable since surface effects are limited in this size range, and have also the same $\tau_{0}$ value. iv) The evolution of $\mu_{0} H_{C H y p}$ with diameter is fitted analytically to determine $\tau_{0}$ and $K_{\text {eff. }}$ v) These two parameters are then injected into numerical simulations to obtain the complete and exact SAR values.

The calculation of $\mu_{0} H_{C H y p}$ as a function of the experimental parameters requires solving numerically:

$$
\mu_{0} H_{C H y p}=0.463 \mu_{0} H_{K}\left(1-\left[\frac{k_{B} T}{K_{e f f} V} \ln \left(\frac{k_{B} T}{4 \mu_{0} H_{C H y p} M_{S} V f \tau_{0}}\right)\right]^{0.8}\right)
$$

Details on the origin and domain of validity of this equation can be found in Supporting Information.

\subsubsection{Results}

The quantitative analysis of the data has been performed for measurements at 55 and at $274 \mathrm{kHz}$ and lead to compatible results. We will focus here on the results obtained at 274 
Submitted to

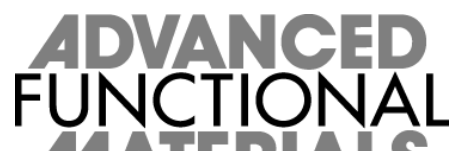

14 A ERALS

$\mathrm{kHz}$, provided that the values of $\mu_{0} H_{\mathrm{CHyp}}$ have a lower error bar due to the higher range of magnetic field used. In Figure 5a, the coercive field $\mu_{0} H_{\text {CHyp }}$ deduced from the highest slope of the $\operatorname{SAR}\left(\mu_{0} H_{\max }\right)$ function is displayed for the four samples. The value for the sample $7(d$ $=27.5 \mathrm{~nm}$ ) is also shown for indication but is not taken into account in the quantitative analysis due to its multi-domain configuration. The size evolution of the coercive field is fitted using Equation (6) with $\tau_{0}$ and $K_{\text {eff }}$ as varying parameters and $\sigma_{\mathrm{S}}=212 \mathrm{~A} \mathrm{~m}^{2} \mathrm{~kg}^{-1}, T=$ $300 \mathrm{~K}$ and $f=274 \mathrm{kHz}$. The best fit of experimental data is obtained for $K_{\text {eff }}=9.1 \times 10^{4} \mathrm{~J} \mathrm{~m}^{-3}$ and $\tau_{0}=5 \times 10^{-11} \mathrm{~s}$ (see Figure 5a). These values have a correct order of magnitude, though $\tau_{0}$ is smaller than the values usually reported for individual MNPs $\left(10^{-9}-10^{-10} \mathrm{~s}\right)$ and $K_{\text {eff }}$ slightly larger than the bulk value. These values were used for numerical simulations and the results compared with experimental data. In Figure $\mathbf{5 b}$, the numerically calculated $S A R$ obtained for the four samples using these parameters are shown. The coercive fields extracted from these curves are shown in Figure 5a as triangles: the points of maximum slope in numerically calculated $\operatorname{SAR}\left(\mu_{0} H_{\max }\right)$ matches the experimental points highlighting the coherency of our method.

The first point of discrepancy between theory and experiments is the amplitude of the SAR. Experimental values as a function of the diameter $\operatorname{SAR}(d)$ are plotted in Figure 5c as square points. Theoretical SAR values, plotted as a dashed line, are well above the experimental values, this for all four samples investigated. To check if this higher value arises from the influence of the size distribution of MNPs, the calculated $\operatorname{SAR}(d)$ function is convoluted with the size distribution deduced from TEM images and plotted as circles in Figure 5c. For large MNPs, the size distribution has a negligible influence, while for small MNPs, the size distribution increases the SAR values. Thus, the size distribution effect can not be held responsible for the smaller experimental SAR values.

Another hypothesis to explain the discrepancy between theory and experiments is the presence of magnetic interactions in the samples whereas the simulations assume noninteracting nanoparticles. In a recent article, D. Serantes et al., have found using numerical simulations of interacting MNPs that ferromagnetic MNPs in dipolar interactions display an enhanced (reduced) SAR at low (high) field, and saturate at a higher field than independent MNPs [29]. For a better comparison on the shape of experimental and theoretical $S A R\left(\mu_{0} H_{\max }\right)$, normalized data are plotted alongside in Figure 5d. As a matter of fact, the data 
Submitted to

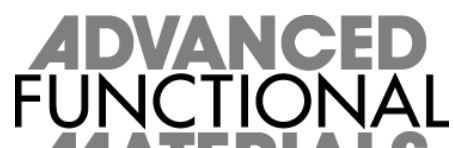

IAARALS

from the samples 4-6 $(d=11.3-19.7 \mathrm{~nm})$ show a striking similarity with the theoretical curves published by D. Serantes et al. As a consequence, we attribute the discrepancies between numerical simulations and experimental data to the presence of magnetic interactions in our samples leading to reduced SAR values and enlarged step width in the $S A R\left(\mu_{0} H_{\max }\right)$ function around the coercive field. This would explain that, even in our optimized samples, $\alpha$ value does not reach the highest possible value (see section 2.2). The next step of optimization would thus consist in elaborating magnetically independent ferromagnetic MNPs of optimized diameter.

\section{Conclusions}

Extensive $\mathrm{MH}$ measurements have been performed on a model system consisting of iron MNPs in a size range going from 5.5 to $27.5 \mathrm{~nm}$. The results have been analysed using state-of-the-art numerical simulations of hysteresis loops and analytical formula. Several features theoretically predicted have been observed for the first time in $\mathrm{MH}$ experiments: i) the increase of coercive field with the size of MNPs ii) the correlation between the amplitude of the coercive field and the amplitude of the SAR iii) the fact that the optimal size depends on the amplitude of the magnetic field. None of these features are predicted by the LRT, though this is the theory that has been the most used so far in MH. These features are however a natural consequence of theories deriving from the Stoner-Wohlfarth model and also appear clearly in numerical simulations. Furthermore the transition toward multi-domain MNPs is clearly detected experimentally by an abrupt drop of both coercive field and SAR, confirming that such MNPs are not the most efficient for $\mathrm{MH}$, the optimized objects being single-domain MNPs.

The variation of coercive field with the diameter of the MNPs is quantitatively reproduced by a simple model of monodisperse non-interacting single-domain MNPs with size-independent $\tau_{0}$ and $K_{\text {eff }}$ values. However, such a model does not correctly reproduce the amplitude of the SAR and its magnetic field dependence. The quantitative analysis we have performed shows that this discrepancy is not due to the effect of size distribution but more likely to the effect of magnetic interactions.

Iron MNPs with optimized properties display hysteresis area values $A=11 \pm 1 \mathrm{~mJ} \mathrm{~g}^{-1}$ at $\mu_{0} H_{\max }=73 \mathrm{mT}$, which is the highest value of the literature so far. The study of their $\alpha$ parameter has shown that they reveal their potential only when large magnetic fields are applied. Keeping a $\mu_{0} H_{\max } f$ product similar to the one used during $\mathrm{MH}$ treatments, they 
Submitted to

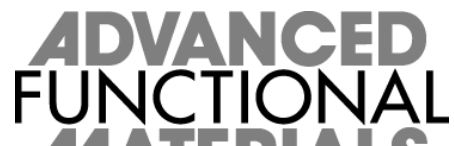

14 A E R A S

display a SAR of $290 \mathrm{~W} \mathrm{~g}^{-1}$. We have seen that this value could be further enhanced since $\alpha$ and thus the SAR could be multiplied by a factor between 3 and 7. The next step of our study will be to reduce the importance of magnetic interactions in our system to check if $\mathrm{MH}$ experiments and numerical simulations would be in quantitative agreement in system of noninteracting MNPs.

Once protected from oxidation, these high-magnetization nano-objects could be useful for several biomedical applications besides magnetic hyperthermia, such as magnetic labelling, drug targeting or contrast enhancement in MRI [30]. For this purpose, a silica layer can be used, as recently patented by our group [31]; this layer could also ensure an easy functionalization of the objects. However, the question of their eventual toxicity should unquestionably be addressed.

\section{Experimental}

Nanoparticles synthesis : All the preparations and syntheses are performed under either an inert atmosphere of argon or a reductive atmosphere of hydrogen using a glove-box and Fisher-Porter bottles. Mesitylene (99\%, VWR Prolabo) was dried over alumina and degassed by three pump-freeze-thaw cycles. Hexadecylamine (HAD, 99\%, Aldrich) was used without purification. Hexadecylammonium chloride was prepared according to published procedure. [32] The $\left\{\mathrm{Fe}\left[\mathrm{N}\left(\mathrm{SiMe}_{3}\right)_{2}\right]_{2}\right\}_{2}$ dimer was brought to NanoMePS and used as-received. Ultrasmall iron(0) NPs were prepared according to published procedure [20].

Synthesis of samples 1 and 2: A solution of palmitic acid (256 mg, $1 \mathrm{mmol}$ for sample 1 and $358 \mathrm{mg}, 1,4 \mathrm{mmols}$ for sample $2 ; 384 \mathrm{mg}, 1.5 \mathrm{mmols})$ in mesitylene (5 ml) was added to a green solution of $\left\{\mathrm{Fe}\left[\mathrm{N}\left(\mathrm{SiMe}_{3}\right)_{2}\right]_{2}\right\}_{2}(376.5 \mathrm{mg}, 0.5 \mathrm{mmol})$ in mesitylene $(10 \mathrm{ml})$ in a Ficher Porter bottle. A solution of hexadecylamine ( $483 \mathrm{mg}, 2 \mathrm{mmols})$ in mesitylene $(5 \mathrm{ml})$ was then added to this mixture. Its color turned to black. The reacting medium was pressurized under 3 bars dihydrogen then heated at $150^{\circ} \mathrm{C}$ for 48 hours. Excess dihydrogen was evacuated and the solution was let to cool at room temperature. The magnetically collected black powder was washed three times with toluene $(15 \mathrm{ml})$. It yielded a material that contained $\mathrm{xx} \%$ and $24.1 \%$ of iron in samples $\mathbf{1}$ and $\mathbf{2}$ respectively. 
Submitted to

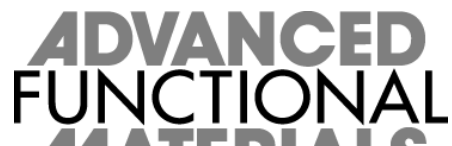

14 A E R A

Synthesis of samples 3 and 4: Hexadecylamonium chloride $(277 \mathrm{mg}, 1 \mathrm{mmol}$ and 415,5 mg, 1,5 mmols) was added to a green solution of $\left\{\mathrm{Fe}\left[\mathrm{N}\left(\mathrm{SiMe}_{3}\right)_{2}\right]_{2}\right\}_{2}(376,5 \mathrm{mg}, 0.5 \mathrm{mmol})$ in mesitylene $(15 \mathrm{ml})$ in a Ficher Porter bottle. The color changed immediately from green to yellow. A solution of hexadecylamine ( $483 \mathrm{mg}, 2 \mathrm{mmols}$ in both cases) in mesitylene (5 ml) was then added to this mixture. Its color turned to black. The reacting medium was heated at $90^{\circ} \mathrm{C}$ for 30 minutes, cooled down to room temperature, then pressurized under 3 bars dihydrogen and heated again at $150^{\circ} \mathrm{C}$ for 48 hours. Excess dihydrogen was evacuated and the solution was let to cool down at room temperature. The magnetically collected black powder was washed three times with toluene $(15 \mathrm{ml})$. It yielded a material that contained $70.4 \%$ and $68.8 \%$ of iron for samples $\mathbf{3}$ and $\mathbf{4}$ respectively.

Synthesis of samples 5, 6 and 7: Ultra-small iron NPs (1 mmol of Fe) were prepared in $10 \mathrm{~mL}$ of mesitylene. Hexadecylammonium chloride $(304,5 \mathrm{mg}, 1.1 \mathrm{mmols}$ for 5; $277 \mathrm{mg}, 1 \mathrm{mmol}$ for 6; $554 \mathrm{mg}, 2 \mathrm{mmols}$ for 7) and hexadecylamine (483 mg, 2 mmols in the three cases) were successively added to the NPs solution. The reacting medium was heated at $90^{\circ} \mathrm{C}$ for 30 minutes, cooled to room temperature, pressurized under 3 bars dihydrogen and then heated again at $150^{\circ} \mathrm{C}$ for 48 hours. Excess dihydrogen was evacuated and the solution was let to cool down at room temperature. The magnetically collected black powder was washed three times with toluene $(15 \mathrm{ml})$. It yielded a material that contained $81.1 \%, 75.6 \%$ and $91.2 \%$ of iron in samples 5, $\mathbf{6}$ and $\mathbf{7}$ respectively.

Magnetic measurements: The magnetization and the coercive field of as-synthesized iron(0) NPs were measured on a Quantum Design Model MPMS 5.5 SQUID magnetometer. The absolute magnetization was deduced from the iron(0) total content determined by Inductively Coupled Plasma - Mass Spectrometry. These studies were carried out on powder samples that were prepared and sealed under an argon atmosphere to preserve the metallic character of iron. Extreme care was taken to avoid oxidation during the transfer to the apparatus.

Hyperthermia measurements: For hyperthermia measurements, a schlenk containing about 12 $\mathrm{mg}$ of powder of Fe NPs and $0.5 \mathrm{ml}$ of mesitylene was filled under inert atmosphere to prevent any oxidation of the NPs. The mesitylene was used as solvent to ensure the solubility of the nanoparticles and because of his high boiling point. The schlenk is then placed in a calorimeter with $1.5 \mathrm{ml}$ of deionised water, the temperature of which was measured. The measurement time was varied between 30 and $100 \mathrm{~s}$, depending on the experimental 
Submitted to

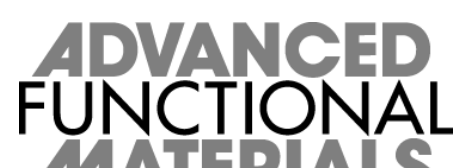

parameters, so that the temperature rise never exceeds $20^{\circ} \mathrm{C}$. The temperature rise at the end of the magnetic field application was always measured after shaking the calorimeter to ensure the temperature homogeneity, which was checked by putting two probes at the top and the bottom of the calorimeter. The temperature rise is determined after this process from the mean slope of the $\Delta T / \Delta t$ function. Then the SAR values were calculated using the expression:

$$
S A R=\frac{\sum_{i} C_{p i} m_{i}}{m_{F e}} \frac{\Delta T}{\Delta t}
$$

Where $C_{\mathrm{pi}}$ and $m_{\mathrm{i}}$ are specific heat capacity and mass for each component $\left(C_{\mathrm{p}}=449 \mathrm{~J} \mathrm{~kg}^{-1} \mathrm{~K}^{-1}\right.$ for Fe NPs, $C_{\mathrm{p}}=1750 \mathrm{~J} \mathrm{~kg}^{-1} \mathrm{~K}^{-1}$ for mesitylene, $C_{\mathrm{p}}=4186 \mathrm{~J} \mathrm{~kg}^{-1} \mathrm{~K}^{-1}$ for water and $C_{\mathrm{p}}=720 \mathrm{~J}$ $\mathrm{kg}^{-1} \mathrm{~K}^{-1}$ for glass), and $m_{\mathrm{Fe}}$ is the mass of the pure Fe MNPs. The mass of glass is put to 2930 mg. Dividing $S A R$ values by the magnetic field frequency lead to the specific losses A.

\section{Acknowledgements}

This work was supported by the InNaBioSante Foundation. Supporting Information is available online from Wiley InterScience or from the author.

Received: ((will be filled in by the editorial staff))

Revised: ((will be filled in by the editorial staff))

Published online: ((will be filled in by the editorial staff))

_[1] P. Moroz, S. K. Jones, and B. N. Gray, Int, J. Hyperthermia 2002 18, 267.

_[2] J. J. W. Lagendijk, Phys. Med. Biol. 2000, 45, R61.

_[3] A. Jordan, R. Scholz, K. Maier-Hauff, M. Johannsen, P. Wust, J. Nadobny, H. Schirra, H. Schmidt, S. Deger, S. Loening, W. Lanksch, R. Felix, J. Magn. Magn. Mater. 2001, 225, 118.

_[4] K. Maier-Hauff, R. Rothe, R. Scholz, U. Gneveckow, P. Wust, B. Thiesen, A. Feussner, A. von Deimling, N. Waldoefner, R. Felix and A. Jordan, J Neurooncol 2007 81, 53

_[5] K. Maier-Hauff, F. Ulrich, D. Nestler, H. Niehoff, P. Wust, B. Thiesen, H. Orawa,V.

Budach and A. Jordan, J. Neurooncol. 2011 103, 317

_[6] M. Johannsen, U. Gneveckow, B. Thiesen, K. Taymoorian, C. H. Cho, N. Waldöfner, R.

Scholz, A. Jordan, S. A. Loening, P. Wust, European Urology 2007 52, 1653

_[7] I.A. Brezovich, Med. Phys. Monogr. 1988, 16, 82.

_[8] P. Wust, U. Gneveckow, M. Johannsen, D. Böhmer, T. Henkel, F. Kahmann, J. Sehouli, R. Felix, J. Ricke, A. Jordan, Int. J. Hyperthermia 2006 22, 673.

_[9] J. Carrey, B. Mehdaoui, M. Respaud, J. Appl. Phys. 2011, 109, 083921

_[10] R. Hergt, R. Hiergeist, M. Zeisberger, G. Glöckl, W. Weitschies, L.P. Ramirez, I. Hilger and W.A. Kaiser, J. Magn. Magn. Mater. 2004, 280, 358. 
_[11] J.-P. Fortin, C. Wilhelm, J. Servais, C. Ménager, J.-C. Bacri and F. Gazeau, J. Am. Chem. Soc. 2007, 129, 2628.

_[12] M. Lévy, C. Wilhelm, J-M. Siaugue, O. Horner, J-C. Bacri and F. Gazeau, J. Phys.: Condens. Mater. 2008, 20, 204133.

_[13] G. F. Goya, E. Lima, A. D. Arelaro, T. Torres, H. R. Rechenberg, L. Rossi, C. Marquina and M. R. Ibarra, IEEE Trans. Magn. 2008, 44, 4444.

_[14] M. Gonzales-Weimuller, M. Zeisberger, K. M. Krishnan, J. Magn. Magn. Mater. 2009, 321, 1947.

_[15] R. Hergt, S. Dutz and M. Zeisberger, Nanotechnology 2010, 21, 015706.

_[16] L.-M. Lacroix, R. Bel-Malaki, J. Carrey, S. Lachaize, G. F. Goya, B. Chaudret, M. Respaud, J. Appl. Phys. 2009, 105, 023911.

_[17] N. A. Usov, J. Appl. Phys. 2010, 107, 123909.

_[18] F. Dumestre, B. Chaudret, C. Amiens, P. Renaud, and P. Fejes, Science 2004, 303, 821.

[19] L.-M. Lacroix, S. Lachaize, A. Falqui, M. Respaud, and B. Chaudret, J. Am. Chem. Soc. 2009, 131, 549.

_[20] L.-M. Lacroix, S. Lachaize, A. Falqui, T. Blon, J. Carrey, and M. Respaud, F.

Dumestre, C. Amiens, O. Margeat, and B. Chaudret, P. Lecante and E. Snoeck, J. Appl. Phys. 2008, 103, 07D521.

_[21] A. Meffre, S. Lachaize, M. Respaud, B. Chaudret, in preparation

_[22] L.-M. Lacroix, J. Carrey, and M. Respaud, Rev. Sci. Instrum. 2008, 79, 093909.

_[23] B. Mehdaoui, A. Meffre, L. M. Lacroix, J. Carrey, S. Lachaize, M. Goujeon, M.

Respaud, B. Chaudret, J. Magn. Magn. Mater. 2010, 322, L49.

_[24] B. Mehdaoui, A. Meffre, L. M. Lacroix, J. Carrey, S. Lachaize, M. Goujeon, M. Respaud, B. Chaudret, J. Appl. Phys. 2010, 107, 09A324.

_[25] R. E. Rosensweig, J. Magn. Magn. Mater. 2002, 252, 370.

_[26] Y. L. Raikher, V. I. Stepanov, R. Perzynski, Physica B 2004, 343, 262.

_[27] W. Rave, K. Fanian, A. Hubert, J. Magn. Magn. Mater. 1998, 190, 332.

[28] E. Snoeck, C. Gatel, L.M. Lacroix, T. Blon, S. Lachaize, J. Carrey, M. Respaud, B. Chaudret, Nanoletters 2008, 8, 4293

_[29] D. Serantes, D. Baldomir, C. Martinez-Boubeta, K. Simeonidis, M. Angelakeris, E. Natividad, M. Castro, A. Mediano, D.-X. Chen, A. Sanchez, LI. Balcells and B. Martínez, J. Appl. Phys. 2010, 108, 073918.

_[30] Q. A. Pankhurst, J. Connolly, S. K. Jones and J Dobson, J. Phys. D: Appl. Phys. 2003, 36 R167-R181

_[31] F. Delpech, C. Nayral, N. El Hawi, WO/2009/071794

_[32] K. Soulantika, A. Maisonnat, M.-C. Fromen, M.-J. Casanove, B. Chaudret, Angew.

Chem. Int. Ed., 2003, 42, 1945. 


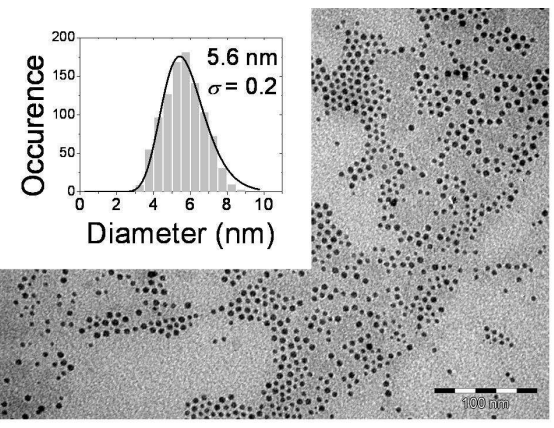

Submitted to
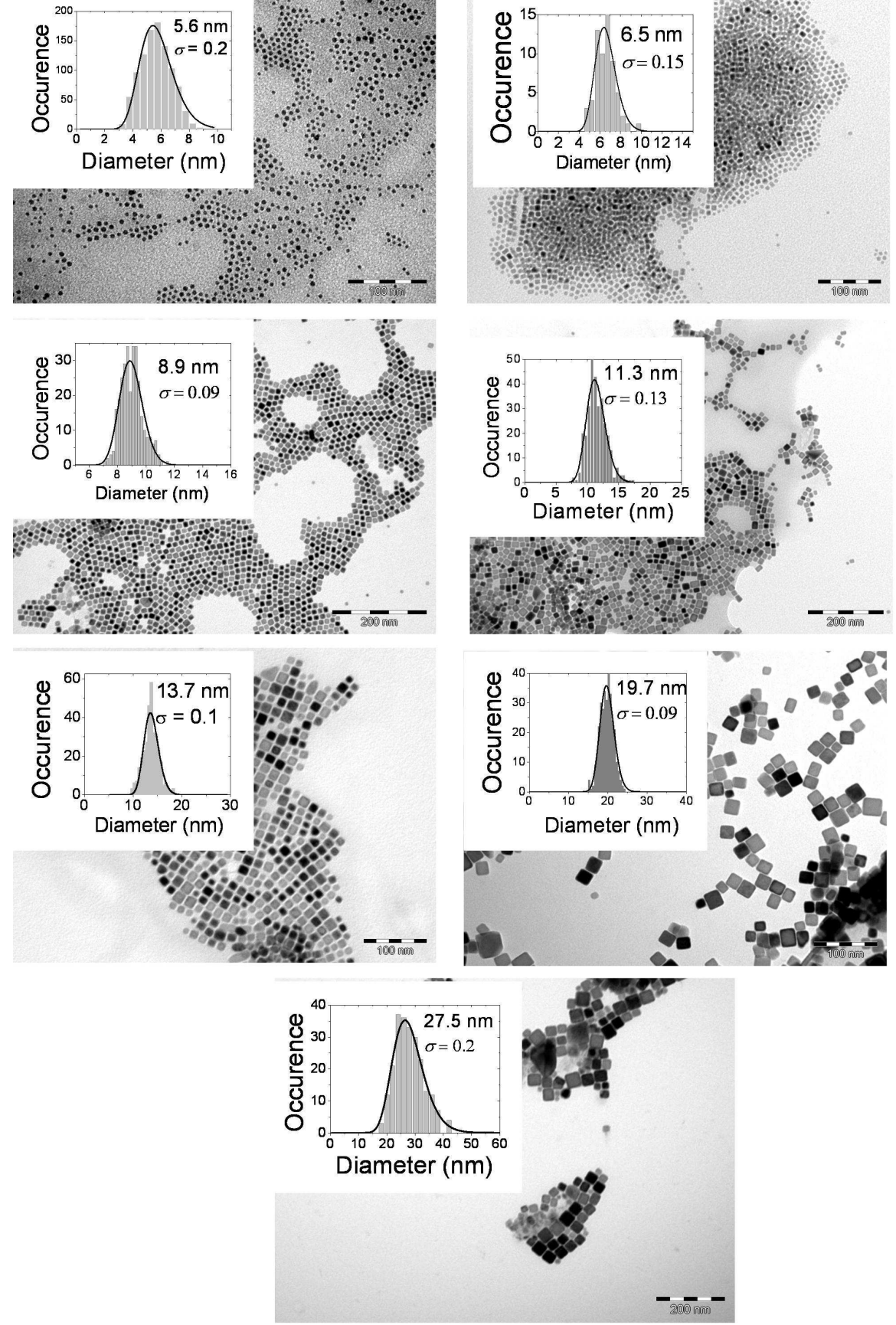

Figure 1. Transmission electron microscopy image of the different samples and corresponding size distribution. The histogram was fitted with a log-normal distribution to estimate the mean particle size $d_{0}$ and the standard deviation $\sigma$. 
Submitted to
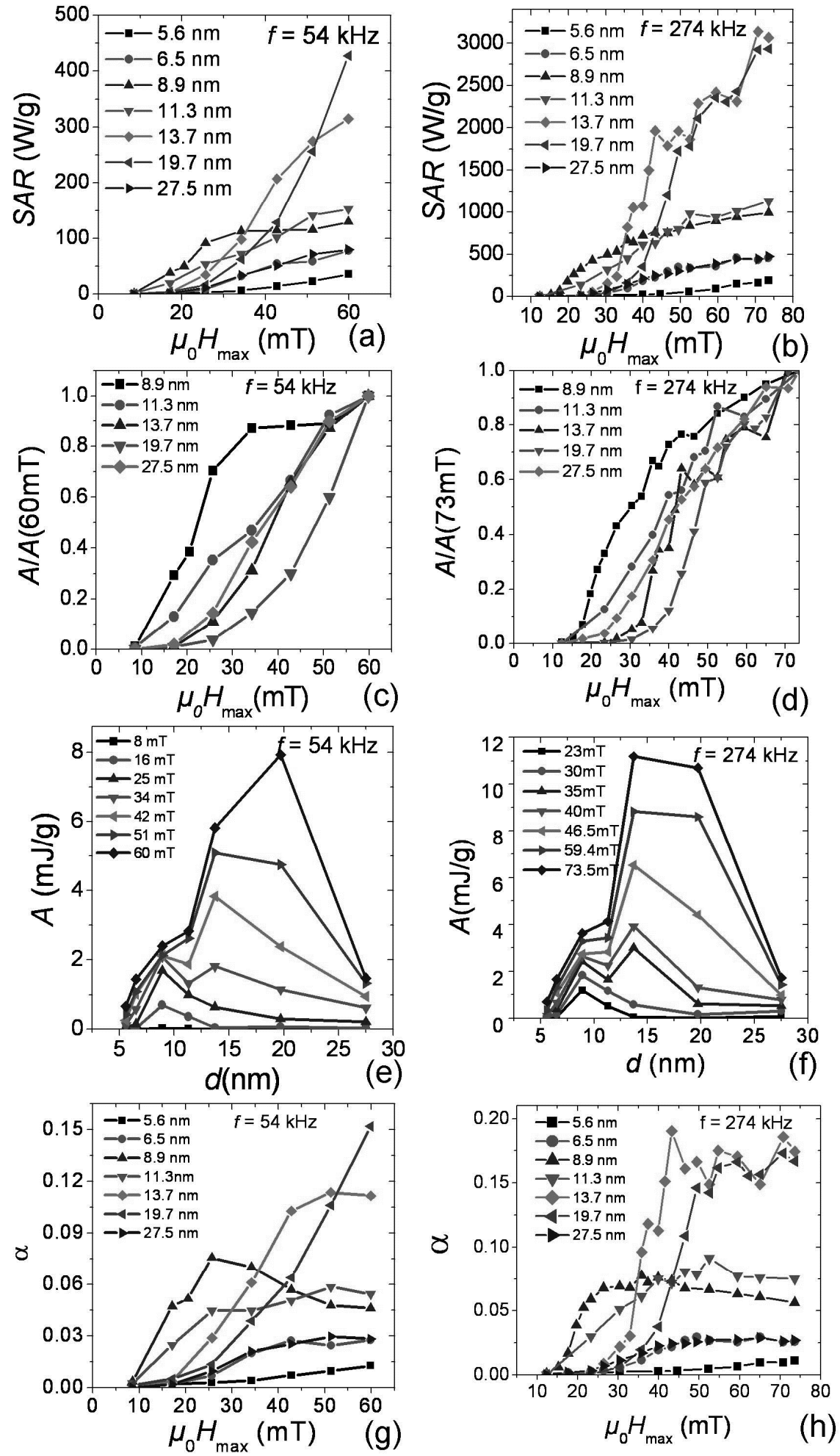

Figure 2. Hyperthermia measurement at frequency $f=54 \mathrm{kHz}$ (left) and $f=274 \mathrm{kHz}$ (right). (a) and (b) Evolution of the SAR as a function of magnetic field for different samples. (c) and (d) Normalized SAR value for NPs ranging between 8.9 and $27.5 \mathrm{~nm}$. (e) and (f) Size dependence of SAR at different magnetic fields. (g) and (h) Data of (a) and (b) modified using Equation (1) to plot the magnetic field dependence of $\alpha$. 


\section{Submitted to}
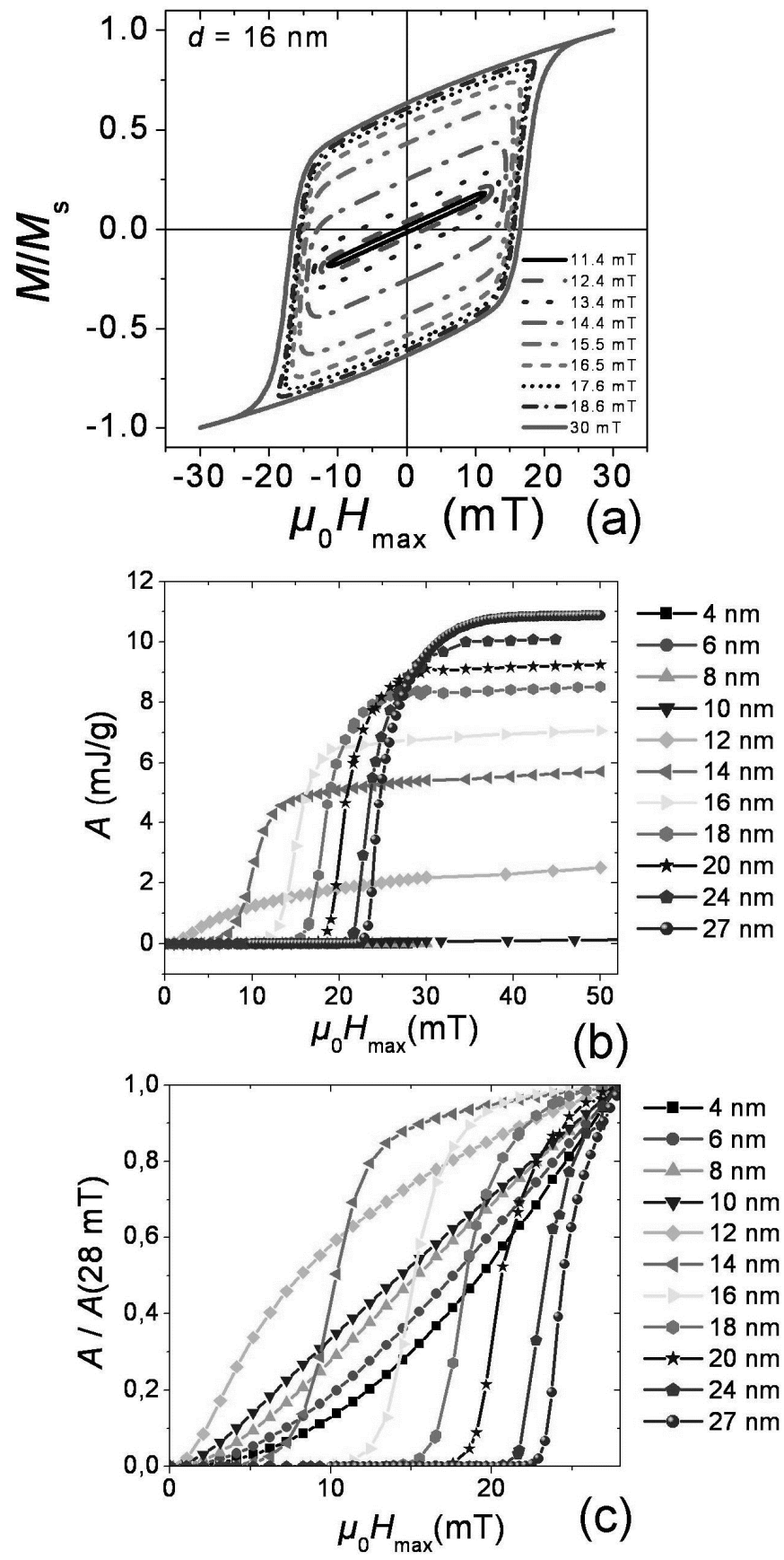

Figure 3. Numerical simulations of the hysteresis area using the parameters of bulk iron. $K_{\text {eff }}$ $=4.8 \times 10^{4} \mathrm{~J} \mathrm{~m}^{-3}, \tau_{0}=5 \times 10^{-11} \mathrm{~s}, M_{\mathrm{S}}=1.7 \mathrm{kA} \mathrm{m}^{-1}, T=300 \mathrm{~K}, f=100 \mathrm{kHz}$. (a) Evolution of the hysteresis loops as a function of the magnetic field for $d=16 \mathrm{~nm}$. (b) Evolution of area as a function of magnetic field for different sizes. (c) Normalized hysteresis area for different size. 
Submitted to
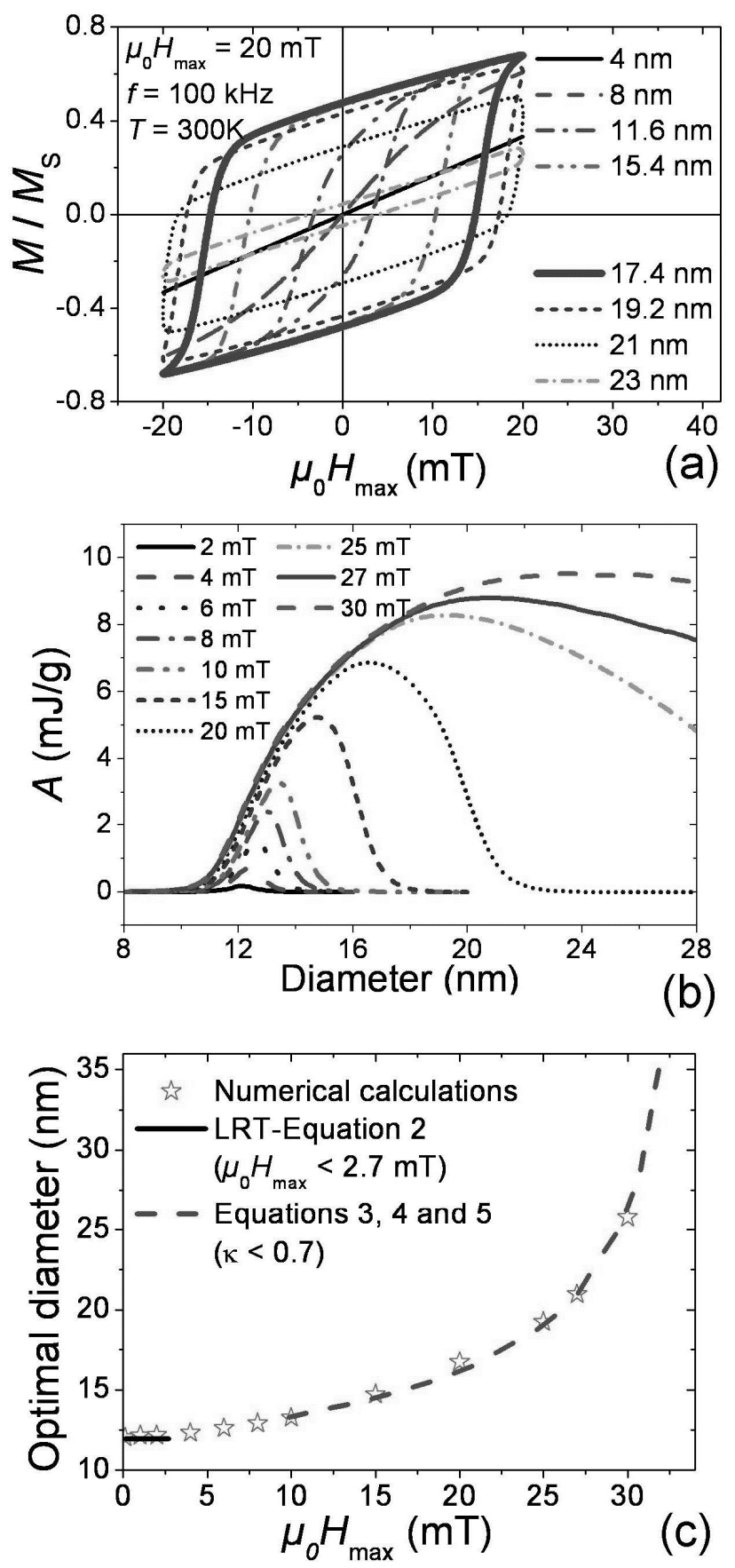

Figure 4. (a) Evolution of the hysteresis loop as a function of size for $\mu_{0} H_{\max }=20 \mathrm{mT}$. (b) Evolution of hysteresis area as a function of size NPs at different magnetic field. (c) Comparison between the optimal diameters calculated analytically using Equation (2) (solid line), Equations (3), (4), (5) (dashed line), and the ones extracted from numerical simulations (star points). 

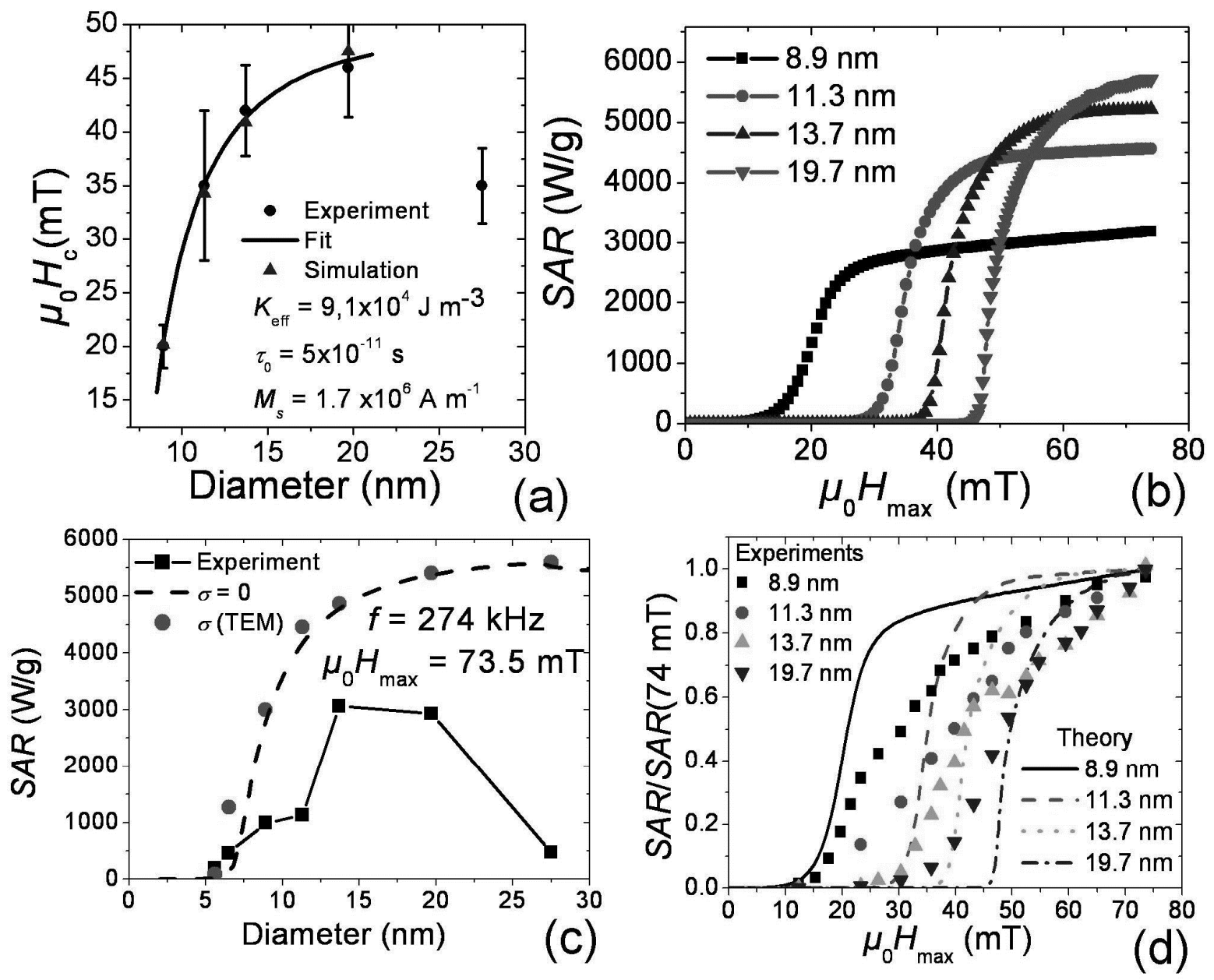

Figure 5. Quantitative analysis of data at $f=274 \mathrm{kHz}$ (a) Evolution of $\mu_{0} H_{\mathrm{CHyp}}$ determined from experiments (round dots), from a fit using Equation (6) (solid line), and from the numerical simulations (triangles). $M_{\mathrm{s}}=1.7 \times 10^{6} \mathrm{~A} \mathrm{~m}^{-1}, T=300 \mathrm{~K}, f=274 \mathrm{kHz}, \tau_{0}=5 \times 10^{-11} \mathrm{~s}$ and $K_{\text {eff }}=9.1 \times 10^{4} \mathrm{~J} \mathrm{~m}^{-3}$. (b) SAR $\left(\mu_{0} H_{\max }\right)$ calculated numerically for four diameters using these parameters. (c) Numerical calculations of the SAR at $\mu_{0} H_{\max }=73.5 \mathrm{mT}$ as a function of the diameter when neglecting the effect of size distribution (dashed line), or using the size distribution deduced from TEM (round dots). Corresponding experimental values (square points). (d) Normalized SAR ( $\left.\mu_{0} H_{\max }\right)$. Dots represents experimental values and lines are results from numerical simulations assuming no size distribution. 
Submitted to

Table 1. Summary of magnetic and hyperthermia properties of the different samples: mean diameter $d_{0}$, standard deviation $\sigma$, saturation magnetization $\sigma_{\mathrm{S}}$, remnant magnetization $\sigma_{\mathrm{R}}$, ratio $\sigma_{\mathrm{R}} / \sigma_{\mathrm{S}}$, coercive field $\mu_{0} H_{\mathrm{C}}$, losses per cycle $A$ at $f=54 \mathrm{kHz}, 60 \mathrm{mT}$, and at $f=274 \mathrm{kHz}$, $73 \mathrm{mT}$, and corresponding $\alpha$ values calculated using Equation (1).

\begin{tabular}{|c|c|c|c|c|c|c|c|c|c|c|}
\hline Samples & $\begin{array}{l}d_{0} \\
{[\mathrm{~nm}]}\end{array}$ & $\sigma$ & 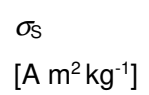 & $\begin{array}{l}\sigma_{\mathrm{R}}\left[\mathrm{A} \mathrm{m}^{2}\right. \\
\left.\mathrm{kg}^{-1}\right]\end{array}$ & $\sigma_{\mathrm{R}} / \sigma_{\mathrm{S}}$ & $\begin{array}{l}\mu_{0} H_{\mathrm{C}} \\
{[\mathrm{mT}]}\end{array}$ & $\begin{array}{l}A(54 \mathrm{kHz}) \\
{\left[\mathrm{mJ} \mathrm{g}^{-1}\right]}\end{array}$ & $\alpha[a]$ & $\begin{array}{l}A(274 \mathrm{kHz}) \\
{\left[\mathrm{mJ} \mathrm{g}^{-1}\right]}\end{array}$ & $\alpha[b]$ \\
\hline 1 & 5.6 & 0.2 & $207 \pm 20$ & 84.8 & 0.41 & 75 & 0.66 & 0.013 & 0.70 & 0.011 \\
\hline 2 & 6.5 & 0.15 & $223 \pm 23$ & 70.0 & 0.31 & 43 & 1.44 & 0.030 & 1.66 & 0.028 \\
\hline 3 & 8.9 & 0.09 & $232 \pm 23$ & 68.5 & 0.30 & 22 & 2.40 & 0.043 & 3.62 & 0.053 \\
\hline 4 & 11.3 & 0.13 & $182 \pm 18$ & 13.0 & 0.07 & 6 & 2.83 & 0.065 & 4.12 & 0.077 \\
\hline 5 & 13.7 & 0.1 & $215 \pm 21$ & 27.5 & 0.16 & 17 & 5.82 & 0.112 & 11.2 & 0.178 \\
\hline 6 & 19.7 & 0.1 & $245 \pm 24$ & 41.0 & 0.16 & 23 & 7.90 & 0.135 & 10.7 & 0.150 \\
\hline 7 & 27.5 & 0.2 & $214 \pm 21$ & 19.1 & 0.10 & 14 & 1.47 & 0.028 & 1.73 & 0.027 \\
\hline
\end{tabular}

[a] $\alpha$ values for measurements at $\mu_{0} H \max =60 \mathrm{mT}$ and $f=54 \mathrm{kHz}$.

[b] $\alpha$ values for measurements at $\mu_{0} H \max =73 \mathrm{mT}$ and $f=274 \mathrm{kHz}$. 


\section{Submitted to

The table of contents entry should be fifty to sixty words long, written in the present tense, and refer to the chosen figure.

Magnetic Nanoparticles, Biomedical Applications, Fe Nanoparticles

By B. Mehdaoui, A. Meffre, J. Carrey*, S. Lachaize, L. M. Lacroix, M. Gougeon, B. Chaudret ,and M. Respaud

Optimal size of nanoparticles for magnetic hyperthermia: A combined theoretical and experimental study

ToC figure ((Please choose one size: $55 \mathrm{~mm}$ broad $\times 50 \mathrm{~mm}$ high or $110 \mathrm{~mm}$ broad $\times 20 \mathrm{~mm}$ high. Please do not use any other dimensions))

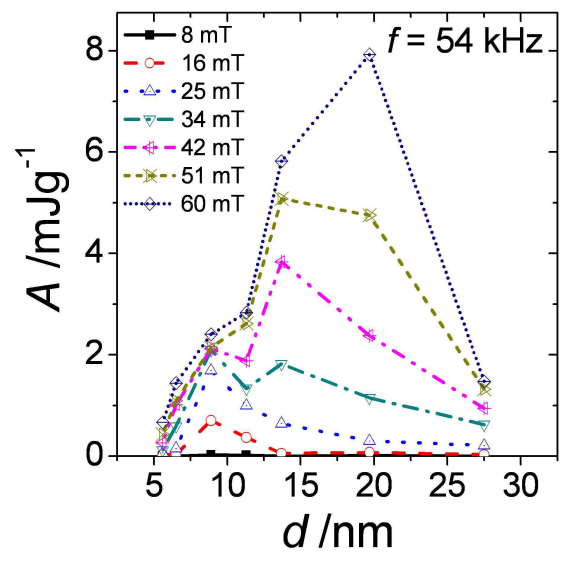

Heating power of metallic iron magnetic nanoparticles whose diameter ranges from $5.5 \mathrm{~nm}$ to $28 \mathrm{~nm}$ is measured in an alternative applied magnetic field of frequency of $54 \mathrm{kHz}$ and of amplitude ranging from 8 to $60 \mathrm{mT}$. The optimal size is visible as a maximum in the curve. Optimized nanoparticles display losses of $8 \mathrm{~mJ} \mathrm{~g}^{-1}$ at this frequency. 